\title{
Perspectives of Malaysian parents on eating out: $A$ qualitative analysis
}

\author{
Suhaila Abdul Ghaffar ${ }^{1,2^{*}}$, Ruzita Abd. Talib $^{1}$ \& Norimah A. Karim ${ }^{1}$ \\ ${ }^{1}$ Nutritional Science Programme, Faculty of Health Science, Universiti Kebangsaan \\ Malaysia, Jalan Raja Muda Abdul Aziz, 50300 Kuala Lumpur; ${ }^{2}$ Institute for Public \\ Health, National Institute of Health, Persiaran Setia Murni, 40170 Shah Alam, \\ Selangor, Malaysia
}

\begin{abstract}
Introduction: The culture of eating out among Malaysians has substantially increased in recent years due to the urbanisation of the society. Frequent eating out has been linked with reduced quality of diet, reduced intake of micronutrients, and increased weight gain. This study aims to explore specific factors on the perceptions and behaviours related to eating out from the perspective of parents. Methods: A qualitative study was carried out among 27 informants from various ethnicities and locations in Peninsular Malaysia. All sessions were audio-recorded, fully transcribed and analysed using thematic content analysis. Results: The main overarching theme that surfaced from this study was the role of parents in shaping the practice of eating out. Time constraints due to parents' work commitments, the availability of various food outlets, increase in family bonding time and food preferences were recurring themes revealed by informants as facilitating factors to eating out. Other emerging themes, such as hygiene of food premises and cost of food, posed as barriers to eating out. Conclusion: These findings offered a better understanding on the eating out practices of a family from the parents' perspective. Information as such could be incorporated in strategies to enhance the promotion of healthy eating and nutrition interventions that aim to inculcate healthy food choices while eating out.
\end{abstract}

Keywords: Eating out, parents, qualitative study, Malaysia

\section{INTRODUCTION}

The culture of eating out is emerging in Malaysia and has undergone a new dimension due to the urbanisation of the society in recent years. Economic growth and urbanisation have caused lifestyle changes among Malaysians where both parents have to work (Ali \& Abdullah 2012). These lifestyle changes include a notable shift in eating culture and are linked to environmental changes such as the increasing number of food outlets in residential areas (Bodicoat et al., 2015).
Another noticeable change is the growth of fast food outlets that makes eating out affordable and convenient. This scenario results in numerous families spending more on eating out (Smith et al., 2013).

Eating out is defined as any food or beverages prepared outside the family home. However, eating out does not include ready-to-eat meals purchased in a supermarket (Lake et al., 2010).

Frequent eating out has been linked with a low-quality diet and weight gain problems (Adams et al., 2015). Foods

\footnotetext{
*Corresponding author: Suhaila Abdul Ghaffar Institute for Public Health, National Institute of Health, Selangor, Malaysia Tel: (6)013-6499551; E-mail: suhaila.aghaffar@gmail.com doi: https://doi.org/10.31246/mjn-2019-0137
} 
consumed away from home are usually high in energy content, a factor that may significantly contribute to excessive energy intake, thereby potentially contributing to obesity (Zeng \& Zeng, 2018). Moreover, eating out is also linked with low consumption of fruits and vegetables (Lachat et al., 2012). Additionally, a study among children aged 2-18 years old in the United States found that fast food outlets contribute more to energy intakes than meals and snacks consumed in schools or day care centres (Powell \& Nguyen, 2013). Therefore, eating out is associated with weight gain among the young generation (Adams et al., 2015). Furthermore, individuals tend to consume more energy and larger portions with lesser vegetables and fruits while eating out (Vandevijvere et al., 2019). Consequently, foods away from home also provide large adverse effects on diet quality and energy intake (Todd et al., 2012). Therefore, individuals who frequently consume meals away from home are also susceptible to noncommunicable diseases (Jilcott et al., 2011).

To understand the eating out practices in a family, the Social Ecological Model (Kilanowski, 2017) illustrates the multilevel influence of individual, social and environmental factors. This study qualitatively explores specific factors on the perceptions and behaviours of parents related to eating out.

\section{MATERIAL AND METHODS}

This study was part of a larger research that focuses on four different target groups with different socio-economic backgrounds. In this study, we focused on parents with adolescents aged 13-17 years old. In-depth interviews guided by semi-structured questions were conducted with 27 parents from multiple locations. Such interviews allowed for in-depth data collection given that the interviewer could probe for explanations from respondents (Fusch \& Ness, 2015).
In addition, insights into the beliefs, attitudes and knowledge pertaining to the informants and their family's eating out experiences and its impact on their health were obtained.

The study was conducted to represent a broad range of population in Peninsular Malaysia, whereby the Northern and Eastern regions were conducted in Pokok Sena, Kedah and Dungun, Terengganu. States in the Central and Southern regions were conducted in Petaling Jaya, Selangor and Muar, Johor. The study population were selected through a purposive sampling method, with preset criteria identified. Access to parents was facilitated by the school teachers, then potential parents were identified by prospective informants via snowball sampling through their acquaintances.

Face-to-face in-depth interviews were conducted with each informant. The inclusion criteria were Malaysian citizens, having either one or more adolescent child(ren) and able to communicate either in English or Malay language (Bahasa Melayu). The interview process was carried out until the information reached a saturation point, whereby no new themes could be generated from the interview session.

\section{Instrument}

Prior to data collection, a research guide was developed to answer the objective of the study. It was based on the grounded theory approach (Charmaz, 2000) and explored the topic of investigation from the informants' perspectives while connecting surfacing findings to the Social Ecological Model. In addition, relevant literature search, the first writer's own experience with guidance from the other two writers and semistructured interview methodology (Bernard, 2016) were used to construct the guide. The guide consisted of a list of questions with useful prompts to encourage informants to respond and provide an in-depth perspective during the interview session. The format 
was a semi-structured research guide with primary and related questions to provide a consistent structure to the questions posed during the interview. The research guide is a mechanism for steering the discussion and enhancing the consistency of data collection. All questions were sequenced from general and comprehensive to specific. The research guide was used during all the interview sessions to ensure consistency in questions asked. However, the in-depth interview sessions were designed to be exploratory and relatively unstructured, allowing new ideas and interesting insights to be pursued (Bernard, 2016). Table 1 displays the primary questions from the research guide.

\section{Study procedure}

A pre-test on the research guide was conducted on three parents to assess the clarity of language, vocabulary and flow of questions. Afterwards, an improved research guide was developed. A pilot test was then conducted on five parents, both in Malay and English languages, to assess the content, estimation of time for each session and identify any practical problems with the responses. The research guide was revised on the basis of feedbacks given by the informants during the pilot study. All study methods and protocols were approved by the Universiti Kebangsaan Malaysia (UKM) Research Ethics Committee prior to study implementation. All informants were provided with a written informed consent form and a subject information sheet for participation and permission to be audio-taped prior to the commencement of the data collection session.

\section{Data collection}

In-depth interviews were conducted individually with informants. A short introduction was provided to explain the confidentiality of the study. Informants were notified that they were free to provide any ideas and feedbacks. Informants were also guaranteed that their information would be treated in a confidential manner, although an audio of each in-depth interview session was recorded for the purpose of analysis. The interview session took an average time of approximately 45 minutes to 1 hour to

Table 1. Research Guide with Primary Topics and Questions

\begin{tabular}{ll}
\hline Primary Topics & Main Questions \\
\hline Introduction & $\begin{array}{l}\text { Could you tell me your personal and family background? } \\
\text { Do you mind sharing your family's eating pattern? } \\
\text { Do you eat outside with your family? } \\
\text { Where do you normally go when eating out?; Which type of eateries } \\
\text { or restaurants? }\end{array}$ \\
Frequency & How frequent do you eat outside with your family members? \\
Cost & How much do you normally spend when eating out? \\
Food Choice & What do you think of the food choices available when eating out? \\
& $\begin{array}{l}\text { How do you find food premises at your neighborhood areas? } \\
\text { What do you think about the price and quality of food? }\end{array}$ \\
Parents' Influences & $\begin{array}{l}\text { May I know the reasons of eating out? } \\
\text { How far does your role as a parent influences decision-making } \\
\text { when eating out? }\end{array}$ \\
& $\begin{array}{l}\text { What type of challenges do you face in practising healthy eating } \\
\text { when eating out? } \\
\text { Do you have any specific facilitators in eating out? } \\
\text { What is your preference when eating out? }\end{array}$ \\
\hline
\end{tabular}


complete. The data collection was carried out from August to November 2016.

\section{Data analysis}

As part of the data analysis, the first writer transcribed all audio recordings to ensure that all sessions were translated into texts to enable systematic analysis (Creswell et al., 2003). Then, the transcribed texts were analysed qualitatively using the inductive thematic analysis approach, Atlas.Ti, and coded via an open coding process. Similar and related themes, sub-themes and main domains were identified (summaries and emergent themes were generated on the basis of transcripts). Two independent reviewers performed content analysis to identify major themes using a combined deductive and inductive approach. To assure content validity within specific themes, inter-rater reliability tests were assessed and found to be acceptable with a Kappa score of 0.80 .

\section{RESULTS}

\section{Informants' characteristics}

This study included a total of 27 informants, aged 43-52 years, and was entirely made up of women (100\%). The majority of participants were Malay $(81 \%)$, followed by Chinese $(15 \%)$ and Indian (4\%). In terms of locality, the informants came from both urban and rural areas. Most participants achieved at least secondary education. Table 2 presents the demographic characteristics of the informants.

Based on the analysed data, predominant perceptions that were derived were summarised under nine themes related to eating out. Each theme was described and representative quotes that illustrated these prevailing perceptions are reported in italics. Responses in local language were translated into English. Table 3 provides the primary answers representative of each theme.
Table 2. Characteristics of the informants

\begin{tabular}{lcc}
\hline Characteristics & $n$ & $\%$ \\
\hline Age (years) & & \\
$\quad$ 40-49 years old & 18 & 67 \\
50 years old and above & 9 & 33 \\
Sex & & \\
$\quad$ Female & 27 & 100 \\
Ethnicity & & \\
$\quad$ Malay & 22 & 81 \\
$\quad$ Chinese & 4 & 15 \\
Indian & 1 & 4 \\
Locality & & \\
$\quad$ Urban & 14 & 52 \\
$\quad$ Rural & 13 & 48 \\
& & \\
Education level & & \\
$\quad$ Secondary & & 22 \\
$\quad$ Tertiary & 6 & 78 \\
\hline
\end{tabular}

Table 3. Themes and sub-themes of eating out

\begin{tabular}{ll}
\hline Theme & Sub-themes \\
\hline Characteristic of & $\begin{array}{l}\text { Frequency of eating out } \\
\text { Types of food outlets }\end{array}$ \\
Individual & $\begin{array}{l}\text { Time scarcity } \\
\text { Food preferences }\end{array}$ \\
& $\begin{array}{l}\text { Cost of foods } \\
\text { Social }\end{array}$ \\
& $\begin{array}{l}\text { Role of parents } \\
\text { Family bonding time } \\
\text { Celebration } / \text { Rewards }^{\ddagger}\end{array}$ \\
& $\begin{array}{l}\text { Availability of food } \\
\text { premises } \\
\text { Environmental } \\
\text { Accessibility to food } \\
\text { sources }\end{array}$ \\
& $\begin{array}{l}\text { Hygiene of food } \\
\text { premises }^{\dagger}\end{array}$ \\
\hline
\end{tabular}

${ }^{\dagger}$ Barriers factors

${ }^{\ddagger}$ Facilitating factors

\section{Eating out characteristics \\ Frequency}

Frequency refers to the number of visits to any food outlet. As noted by the informants, the frequency of eating out was based on family decision. Several 
families frequently ate at food outlets whereas others frequently ate at home.

Hmmm... No, not frequently. If we can (eat out) once a month, that's considered a lot. (Informant 24, Mother)

Roughly four to five times in a month. (Informant 8, Mother)

I don't normally buy food outside. Will cook. (Informant 20, Mother)

Types of food outlets

In general, informants revealed that they have access to various types of food outlets in their neighbourhood, which included fast food outlets, food stalls and restaurants.

Sometimes Chinese restaurant, (sometimes) Thai restaurant... because we can choose what we want to order. (Informant 24, Mother)

I can say that we often go to fast food outlets and the restaurants by the roadside. (Informant 21, Mother)

We seldom go to hawker stalls. But sometimes, (we'll go because) we want to introduce variety of foods (to the children) with different environment so that they will experience eating at places without air-conditioning. (Informant 27, Mother)

\section{Factors influencing the practice of eating out}

Multifaceted themes regarding eating out behaviour from the perspective of parents were identified based on the Social Ecological Model. The themes could be categorised under different factors, such as individual, social and environmental. Based on the findings of the study, the themes were then classified as facilitators and barriers for eating out practice.

Parents' role

Parents play a vital role in the eating out behaviour of family members. The majority of informants said that they were aware of their role in determining the food intake and physical development of their children.
Normally, when they want to eat pizza, instead of taking them out, I'll try to make it myself. (Informant 26, Mother)
Yes, that's quite often. Sometimes pizza, sometimes KFC. The children will request for it. But it's up to us whether or not to take them there. (Informant 21, Mother)
So... the food that I decide to buy, the food I bring back home, that's my decision. You are what you eat... (Informant 27, Mother)

Time scarcity

Most of the informants highlighted time scarcity due to work commitments as a facilitating factor to eating out. Most parents reported that they did not have the time to prepare food at home for their family.

When both of us are working... coincidentally I go back late... and my husband as well. So there's not enough time to prepare food at home. (Informant 18, Mother)

Usually when busy working... not enough time to prepare. (Informant 9, Mother)

Yes, time and commitment. When I reach home, it's already $4 \mathrm{pm} . .$. sometimes it's 5pm. So we are too tired then. (Informant 21, Mother) 
Food preferences

Food preferences vary between individuals. One facilitating factor influencing the practice of eating out is the accessibility to different types of foods. In addition, some families might want to try new food choices away from home.

Yes, we normally go to western food due to their (children) preference. (Informant 8, Mother)

Sometimes we will give the kids fast food, even though we know it's not really healthy...but since the kids requested... and we also feel like having it as well... (Informant 18, Mother)

And my children always want to try new food. (Informant, 20, Mother)

\section{Family/Bonding time}

Another facilitating factor to eating out is the parents' desire to spend quality time with their children. Many informants highlighted the fact that eating outside was also considered as family time. Parents reported that they preferred eating away from home because everyone could order what they wanted to eat.

Normally on weekend, we can all go together and I can rest... because on weekdays, everybody is busy. Rushing for tuition and to do this and that, and it's very jam-packed to go out. (Informant 8, Mother)

My husband always says that we often gather together on Sundays...so we'll eat outside. When the children comes back from the boarding school, if we can't have breakfast together, probably we can do lunch instead. (Informant 11, Mother)
Celebration/Rewards

Family events, such as personal achievements, birthdays and other special occasions were identified as a facilitator to eating out as family members can celebrate together at the food outlet.

Sometimes we celebrate (the children's) birthdays outside... or if there's any occasions. (Informant 4, Mother)

I want to show my appreciation to them. So I will let them choose whatever (food) they want. (Informant 28, Mother)

\section{Sometimes when there are visitors coming over, like relatives. Then we'll go out for meals. (Informant 24, Mother)}

Availability

Informants also reported that environmental factors, such as the availability of various types of food premises around the neighbourhood as a facilitating factor to eating out.

...the environment made it easy for the kids to notice the shops, the fast food outlets and the newly opened restaurants. So there are many types of foods nowadays. (Informant 20, Mother)

Accessibility

Several informants reported that accessibility to various food sources helped them to have more options or alternative food types. In fact, a cluster of services provided by food outlets such as drive-through, extended operation hours and delivery services encouraged them to eat out. 
... just close to my house...

and there are even a few more (outlets) in this district (Informant 5, Mother)

Yes...because it's more convenient due to its operation hours. (Informant 24, Mother)

But now they have delivery services. So, if we don't (want to) go out, sometimes we'll just order delivery...whenever we don't feel like having rice. (Informant 20, Mother)

Cost

Informants reported that cost was a significant barrier to eating out. Majority admitted that the cost of eating out was higher as compared to eating at home. For informants with many members in their household, eating out incurred higher costs and thus demotivated them from such practice.

Actually, it's not worth it. Normally when eating out, I'll say, 'with RM100...I can buy raw ingredients such as fresh meat, seafood... and cook (for the family) for a week'. (Informant 4, Mother)

I don't normally...we don't really (eat out). We don't spend a lot... because if we feel like eating seafood such as lobsters or crabs... because we are quite a big family... we are aware that eating out will be expensive. So, we will end up ordering just fish and omelette. (Informant 12, Mother)

Too expensive. (Informant 19, Mother)

\section{Hygiene}

A few informants were concerned with the hygiene of the premises while eating out. Outlets with poor hygiene practices became one of the barriers to eating out. Although most of the parents preferred to patron food outlets with good hygiene practices, sometimes these were hard to find.

...we normally prefer the restaurants that are clean, but there are not many options. (Informant 24, Mother)

Eating at home will be healthier, right? But when we eat outside, I can't dictate the cleanliness of the food. (Informant 14, Mother)

\section{DISCUSSION}

This study investigated the factors that influenced family members to eat out, based on the parents' perspective. The results revealed that eating out is an alternative to preparing food at home. Majority of the informants practised this eating behaviour frequently, at least once a month. Parallel to family practices, eating out was considered part of a family's activity regardless of locality (urban or rural) and socioeconomic background of informants. However, those residing in urban areas were found to eat out more frequently than those in rural areas. The findings were generally aligned with studies carried out by Cynthia et al. (2013) and Ali \& Abdullah (2012), who reported that Malaysians, especially those in urban areas, opt for fast and easy meals to suit their hectic lifestyle. In contradiction, a study in the United States showed that eating out practices were more common among the lower socio-economic group and minority populations located in the sub-urban areas (Nguyen \& Powell, 2003).

An overarching theme that surfaced was about the fundamental role parents play in the choices and purchases of foods when eating out. Parental behaviours are crucial in stimulating healthy eating 
practices in their children. This was in line with earlier findings by Kasparian et al. (2017) where parents play a significant role in their family's eating out practices and also decide whether to facilitate or discourage eating out. Another study carried out in Melbourne and Geelong, Australia, revealed that parents are likely to be essential mediators of fast food consumption (Timperio et al., 2009). As a result, parents can influence their children's eating behaviour through specific feeding practices and parenting style.

Besides, this study highlighted various factors that facilitated and hindered the habit of eating out in a family. The facilitating factors were time constraints, food preferences, bonding time among family members, and the availability and accessibility of food outlets. One factor that was largely responsible for eating out practices was time constraint due to parents' work commitments and having no time to prepare food for other family members. This was similar to other study carried by Mancino et al. (2009), which showed a positive relationship between a woman's opportunity and cost of time, whereby working mothers have limited time to prepare food for the family.

From the findings, informants also reported that the food preferences of their children played a role in facilitating eating out. A revolution on consumer tastes, especially in children, has been observed. Cooking programmes, overseas travel, and growing cultural and ethnic diversity (Habib et al., 2011) have broadened the experience of eating out by wanting to explore new cuisines, which can often be found outside of home.

The perception of eating out as a treat was one of the most frequently mentioned factors by parents, which aligned with the study of Eck et al. (2019). Several informants mentioned that important family events, such as birthday celebrations, served as treats or rewards for their children. All these celebrations were identified as another factor encouraging eating out practices in families (Anderson, 2002).

As noted by the informants, the availability of food premises led to more food options on offer, which encouraged the behaviour of eating out. Food premises can range from street stalls to fast food outlets and other restaurants. This might be due to the improved socioeconomic status of Malaysians, which has encouraged the mushrooming of food outlets in the neighbourhood. In addition, the increased number of food premises and fast food outlets near residential areas provided accessibility and convenience to the informants (Belon et al., 2016). Day \& Pearce (2011) reported that easy access to food outlets within the neighbourhood increasingly facilitate eating out. The findings also revealed that delivery services from nearby restaurants and fast food outlets provided motivation for informants to eat out. This is parallel with Xin (2016), who reported that the commercial foodservice industry now provide extra services and facilities to its customer. Therefore, the close distance between home and food outlets, coupled with delivery services, increased the accessibility of eating out practices.

Meanwhile, this study has also identified barriers to eating out. These barriers included the cost of foods and hygiene of food premises. Informants mentioned that foods prepared outside of home were more costly compared with home-cooked foods. They perceived that eating out was costly, particularly for those with many family members. Therefore, the cost of foods was one of the crucial factors to consider when eating out. This finding supported the studies by Scozzafava (2017), which revealed that the price of foods, coupled with the quality of service, was the most important element in eating out. Furthermore, other informants claimed that eating at home was associated with 
cost effectiveness and also inculcated healthy eating practices because they can choose the ingredients for their foods. Nearly all informants identified hygiene standard of food outlets as a deterrent. Prentice (2003) revealed that foods outside of homes might be associated with poor hygiene during preparation, storage and handling that may lead to diseases.

The present study has several limitations which should be noted. Firstly, this qualitative study was solely dependent on self-reporting, which might not reflect the actual scenario of eating out practices among Malaysian parents. In addition, as the current study only involved female parents, the results are not generalisable to male parents.

Despite these limitations, this study represented parents of adolescents from multi-socioeconomic backgrounds and from various locations. Therefore, the results have established the eating out practices among parents from different backgrounds. Also, the indepth interview approach provided factors related to eating out as perceived by parents, thus would be useful to the uptake of targeted interventions or evidence-based guidelines. These insights and contributions might help to employ a quantitative method in measuring the constructs revealed by the respondents of this study.

\section{CONCLUSION}

In conclusion, numerous factors influenced the family practice of eating out. The facilitating factors included time constraint, availability and accessibility of food outlets, family bonding time and food choices or preferences. The two barriers to eating out were hygiene and cost. The results of our study showed that the facilitating factors of eating out appeared to be stronger than the barriers, as mentioned by the informants. Therefore, informants' feedbacks provided essential input for further studies to understand the eating practices of family members and inculcate healthy practices when eating out.

\section{Acknowledgement}

We would like to express our sincerest gratitude to all respondents who have voluntarily participated in this study.

\section{Authors' contributions}

SAG, substantial contributions to conception and design, data acquisition, analysis and/or interpretation; and drafting the article or revising it critically for important intellectual content; RAT, final approval of the version to be published; NAK, final approval of the version to be published.

\section{Conflict of interest}

The authors declare that there was no conflict of interest in the publication of this paper.

\section{References}

Adams J, Goffe L, Brown T, Lake AA, Summerbell C, White M, Wrieden W \& Adamson AJ (2015). Frequency and socio-demographic correlates of eating meals out and take-away meals at home: cross-sectional analysis of the UK national diet and nutrition survey, waves 1-4 (2008-12). Int J Behav Nutr 12:51. doi:10.1186/s12966-0150210-8.

Ali N \& Abdullah M (2012). The food consumption and eating behaviour of Malaysian urbanites. issues and concerns. Malays $J$ Soc Space 3(1):44-53.

Anderson A (2002). Alan Warde \& Lydia Martens: Eating out: social differentiation, consumption and pleasure. J Consum Policy 25(3-4):457460. doi:10.1023/A:1020373609615.

Belon AP, Nieuwendyk LM, Vallianatos $H$ \& Nykiforuk CIJ (2016). Perceived community environmental influences on eating behaviors: A Photovoice analysis. Soc Sci Med 171:18-29. doi:10.1016/j.socscimed.2016.11.004.

Bernard HR, Wutich A \& Ryan GW (2016). Analyzing qualitative data: Systematic approaches. SAGE publications.

Bodicoat DH, Carter P, Comber A, Edwardson C, Gray LJ, Hill S, Webb D, Yates T, Davies MJ $\&$ Khunti K (2015). Is the number of fast-food outlets in the neighbourhood related to screendetected type 2 diabetes mellitus and associated risk factors? Public Health Nutr. 18(9):1698705. doi: $10.1017 / \mathrm{S} 1368980014002316$. 
Charmaz K (2000). Grounded theory: Objectivist and constructivist methods. Handbook of qualitative research. Sage Publications, Inc.

Creswell JW, Plano Clark VL, Gutman ML \& Hanson EW (2003). Advanced Mixed Methods Research Designs. In VL Plano Clark \& JW Creswell (eds.). The Mixed Methods Reader (pp.159-196). SAGE Publications, California.

Cynthia J, Zalilah MS \& Lim MY (2013). Relationship between family meals away from home and nutritional status of adolescents. Mal J Nutr 19(1):25-35.

Day PL \& Pearce J (2011). Obesity-promoting food environments and the spatial clustering of food outlets around schools. Am J Prev Med 40(2):113-121.

Eck KM, Delaney C, Olfert MD, Hagedorn RL, Leary MP, Santella ME, Clark RL, Famodu OA, Shelnutt KP \& Byrd-Bredbenner C (2019). Parents' and kids' eating away from home cognitions. Brit Food J 121(5):1168-1182. doi:10.1108/BFJ-07-2018-0431.

Fusch PI \& Ness LR (2015). Are we there yet? Data saturation in qualitative research. The Qualitative Report 20(9):1408-1416.

Habib FQ, Abu Dardak R \& Zakaria S (2011). Consumers' preference and consumption towards fast food: Evidences from Malaysia. Business Management Quarterly Review 2(1): 14-27.

Jilcott SB, Wade S, McGuirt JT, Wu Q, Lazorick S \& Moore JB (2011). The association between the food environment and weight status among eastern North Carolina youth. Public Health Nutr 14(09):1610-1617. doi:10.1017/ S1368980011000668.

Kasparian M, Mann G, Serrano EL \& Farris AR (2017). Parenting practices toward food and children's behavior: Eating away from home versus at home. Appetite 114:194-199. doi:10.1016/j.appet.2017.03.045.

Kilanowski JF (2017). Breadth of the SocioEcological Model. J Agromedicine 22(4):295297. doi:10.1080/1059924X.2017.1358971.

Lachat C, Nago E, Verstraeten R, Roberfroid D, Van Camp J \& Kolsteren P (2012). Eating out of home and its association with dietary intake: a systematic review of the evidence. Obes Rev 13(4):329-46. doi:10.1111/j.1467789X.2011.00953.x.

Lake AA, Burgoine T, Greenhalgh F, Stamp E \& Tyrrell R (2010). The foodscape: Classification and field validation of secondary data sources. Health Place 16(4):666-673.
Mancino L, Todd J \& Lin B (2009). Separating what we eat from where: Measuring the effect of food away from home on diet quality. Food Policy 34(6):557-562. doi:10.1016/j. foodpol.2009.09.003.

Nguyen BT \& Powell LM (2003). The impact of restaurant consumption among US adults: effects on energy and nutrient intakes. Public Health Nutr (11):2445-2452. doi:10.1017/ S1368980014001153.

Powell LM \& Nguyen BT (2013). Fast-food and full-service restaurant consumption among children and adolescents: effect on energy, beverage, and nutrient intake. JAMA Pediatrics 167(1):14-20. doi:10.1001/ jamapediatrics.2013.417.

Prentice AM \& Jebb SA (2003). Fast foods, energy density and obesity: a possible mechanistic link. Obesity reviews 4(4):187-194.

Scozzafava G, Contini C, Romano C \& Casini L (2017). Eating out: which restaurant to choose? British Food Journal.

Smith D, Cummins S, Clark C \& Stansfeld S (2013). Does the local food environment around schools affect diet? Longitudinal associations in adolescents attending secondary schools in East London. BMC Public Health 13(1):70. doi:10.1186/1471-2458-13-70.

Timperio AF, Ball K, Roberts R, Andrianopoulos N \& Crawford DA (2009). Childrens takeaway and fast-food intakes: Associations with the neighbourhood food environment. Public Health Nutr 12(10):1960-1964. doi:10.1017/ S1368980009004959.

Todd JE, Mancino L \& Lin B-H (2012). The Impact of Food Away from Home on Adult Diet Quality. USDA-ERS Economic Research Report Paper No. 90. doi:10.2139/ssrn.1557129.

Vandevijvere S, Lachat C, Kolsteren P \& Van Oyen $\mathrm{H}$ (2019). Eating out of home in Belgium: current situation and policy implications. $\mathrm{Br} J$ Nutr 102:921-928. doi:10.1017/ S0007114509311745.

Worsfold D \& Worsfold PM (2007). How clean is that cafe? Online hygiene inspection reports for consumers. $J$ Foodservice 18(3):93-100. doi:10.1111/j.1745-4506.2007.00051.x.

Xin W (2016). Strategic Marketing Management of Food Delivery. Bachelor's Thesis. HAMK University of Applied Sciences, Finland.

Zeng Q \& Zeng Y (2018). Eating out and getting fat? A comparative study between urban and rural China. Appetite 120:409-415. doi:10.1016/j. appet.2017.09.027. 